Article

\title{
Robust Audio Content Classification Using Hybrid-Based SMD and Entropy-Based VAD
}

\author{
Kun-Ching Wang $\mathbb{D}$
}

Department of Information Technology \& Communication, Shih Chien University, No. 200, University Rd, Neimen Shiang, Kaohsiung 845, Taiwan; kunching.wang@gmail.com

Received: 16 December 2019; Accepted: 28 January 2020; Published: 6 February 2020

check for updates

\begin{abstract}
A robust approach for the application of audio content classification (ACC) is proposed in this paper, especially in variable noise-level conditions. We know that speech, music, and background noise (also called silence) are usually mixed in the noisy audio signal. Based on the findings, we propose a hierarchical ACC approach consisting of three parts: voice activity detection (VAD), speech/music discrimination (SMD), and post-processing. First, entropy-based VAD is successfully used to segment input signal into noisy audio and noise even if variable-noise level is happening. The determinations of one-dimensional (1D)-subband energy information (1D-SEI) and 2D-textural image information (2D-TII) are then formed as a hybrid feature set. The hybrid-based SMD is achieved because the hybrid feature set is input into the classification of the support vector machine (SVM). Finally, a rule-based post-processing of segments is utilized to smoothly determine the output of the ACC system. The noisy audio is successfully classified into noise, speech, and music. Experimental results show that the hierarchical ACC system using hybrid feature-based SMD and entropy-based VAD is successfully evaluated against three available datasets and is comparable with existing methods even in a variable noise-level environment. In addition, our test results with the VAD scheme and hybrid features also shows that the proposed architecture increases the performance of audio content discrimination.
\end{abstract}

Keywords: audio content classification; spectral entropy; voice activity detection; speech/music discrimination; wavelet packet; support vector machine

\section{Introduction}

With the rapid growth of information technology, multimedia management is a very crucial task. Multimedia is needed to classify different data types for efficient accessing and/or retrieving. Knowing how to build a management of multimedia information for $\mathrm{AV}$ (audio/video) indexing and retrieval is becoming extremely important. In the field of $\mathrm{AV}$ indexing and retrieval, the speech/music discrimination (SMD) is a very crucial task for the audio content classification (ACC) system or general audio detection and classification (GADC) [1-18]. In recently, the SMD literatures have been presented in different application [19-24] and closely related to retrieval of audio content indexing [20]. In general, audio feature extraction and audio segmentation are two main parts of a content-based classifier. Different features are presented to describe audio data. These features are mainly categories characteristic of time-domain and frequency-domain. In terms of feature extraction, the very common time-domain features are short-time energy (STE) $[25,26]$ and the zero-crossing rate (ZCR) $[27,28]$. Signal energy [29-31], fundamental frequency [32], Mel frequency cepstral coefficients (MFCC) [19,33,34] are the most used frequency-domain features. Recently, a few studies focused on speech and song/music discrimination [35-37]. Some features such as loudness and sharpness have been incorporated in the human hearing process to describe sounds [38,39]. In a study by [40], a novel feature extraction method based on the visual signature extraction is presented. The well-known "spectrogram reading" 
is regarded as visual information and displays the representation of time-frequency. In the visual domain, the representation of time-frequency successfully stands for the audio signal pattern $[40,41]$. In addition, various techniques of audio classification are used for characterizing music signals, such as threshold-based methods or combining the string tokenization method and data mining technique [42]. Neural network [43], clustering [44], and k-nearest neighbor (k-NN) are used for speech/music classification, and the decision is made based on a heuristic-based approach [45]. In [46], the decision relies on the k-NN for classification by using perceptually weighted Euclidean distance. Gaussian mixture models (GMM) [47], support vector machine (SVM) [48], and fuzzy-rule [49] are also used for speech/music classification. Such new trends include temporal feature integration and classifiers aggregation [8-15], novelty audio detection and bimodal segmentation [7,9,16], and deep learning $[5,17]$. In recent years deep learning algorithms have been successfully used to solve numerous speech/noise classification problems, especially the development of deep convolutional neural networks without any need for careful feature selection [50,51]. However, deep neural networks are generally known to be more computationally expensive and slower than other more conventional methods [52]. Apart from the above, innovative techniques utilizing one-class classifiers, perceptual wavelet-cepstral parameters, hierarchical/multi-resolution thresholding, and other adaptive detection mechanisms were recently reported [7,9-11].

Up to now, in a real-life environment, the problem of a variable-noise level environment is not considered for the above-mentioned works. To alleviate this problem, the robust spectral entropy-based scheme of voice-activity detection (VAD) which distinguishes speech and non-speech segments from the incoming audio signal is combined with the utilized SMD approach as a front-end of the proposed system of ACC application. Especially for the VAD case, the idea of using spectral entropy and other related parameters that monitor spectral variability or flatness has been used for many years $[1,3,4]$. Our previous research article [53] proved that spectral entropy-based VAD can be successfully applied to a variable noise-level environment. In addition, the differences on the sound spectrogram between music and speech are significant. In music, the spectrum's peak tends to change relatively slowly even though music is played with various tempos as shown in Figure 1. On the contrary, shorter durations occur in speech sound events. We know that the spectral envelope of speech varies more frequently than the spectral envelope of music. Consequently, the rate of change of the spectral envelope (or called texture diversity) is one of the valid features for characterizing the differences between speech and music. This type of texture diversity suggests that perceptual wavelet analysis on a spectrogram will generate highly discriminate features for audio discrimination. Texture diversity is also regarded as 2D textual image information on a spectrogram and was successfully applied in studies by [54] and [55].

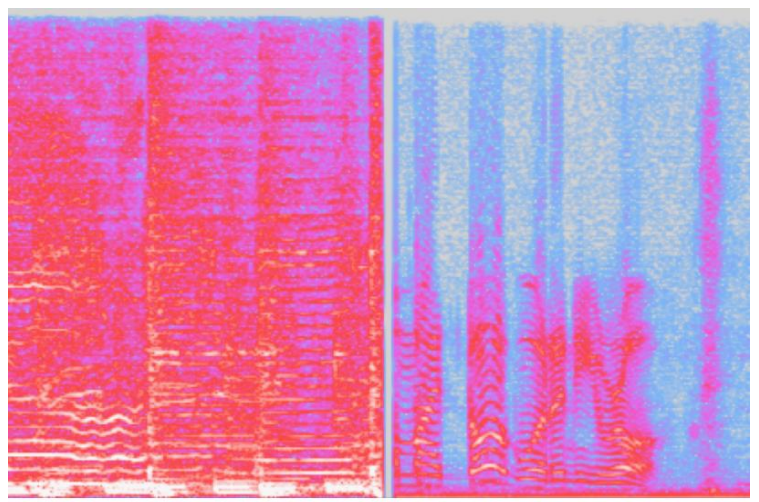

(a) Music Signal

(b) Speech Signal

Figure 1. Sound spectrogram for music and speech. (a) Spectrogram on music and (b) spectrogram on speech.

Extended from our previous work [56], a hierarchical scheme of the ACC system is proposed in this paper. In general, audio hierarchically categorizes silence/background noise, various music 
genres, and speech signals. As a result, a three-stage scheme involving speech, music, and other is adopted herein [9]. In the first stage of the proposed ACC, the incoming audio signal is pre-emphasized and partitioned. Next, the scheme of VAD is utilized with the Mel-scale spectral entropy to classify the emphasized audio signal into silence segments and non-silence segments. In the second stage, the SMD approach comprises of the extraction of hybrid features and SVM-based classification. A novel technique of hybrid feature extraction is derived from wavelet-spectrogram textual information and energy information to obtain a set of features including the 1D subband energy information (1D-SEI) and 2D texture image information (2D-TII).

In order to extract the 2D-TII parameter, we first generated the spectrogram in grayscale. Then, the local information was captured by zoning the range from $0 \mathrm{kHz}$ to $4 \mathrm{kHz}$ in order to characterize the discrimination between speech and music [57]. This is so the 2D-texture information [54] can be analyzed upon the wavelet-spectrogram. Next, the 2D-TII parameter is accurately obtained by using Laws' mask through 2D-perceptual wavelet packet transform (PWPT). Consequently, we let three hybrid feature inputs into an SVM classifier. During the second stage, the noisy audio segments are classified into speech segments and music segments. In the third stage we improved the discrimination accuracy, and a rule-based post-processing method was applied to reflect the continuity of audio data in time.

This paper is organized as follows. In Section 2, we introduce the proposed approach of the three-stage ACC. The approach includes three main stages: pre-processing/VAD, SMD, and post-processing. The VAD uses the measure of band-spectral entropy to distinguish non-noise segments (noisy audio segment) from noise segments (silence). Section 3 presents the hybrid-based SMD algorithm. The hybrid features include 1D subband energy information (1D-SEI) and 2D texture image information (2D-TII). Through the combination of $1 \mathrm{D}$ signal processing and $2 \mathrm{D}$ image processing, the hybrid features characterize the discrimination between speech and music. In Section 4, the rule-based post-processing is presented to improve the segmentation results in different noise types and levels. Finally, the experiments and results are presented in Section 5. In this section, the evaluation of the proposed ACC approach is performed on well-known speech and music databases (e.g., GTZAN dataset) at well-defined signal-to-noise ratio (SNR) levels. Section 6 provides the discussion and conclusions.

\section{The Architecture of Hierarchical Based ACC Approach}

Figure 2 shows the block diagram of the audio content classification (ACC) system, which is divided into three main stages: pre-processing/voice activity detection (VAD), speech/music discrimination (SMD), and rule-based post-processing. The details are described below.

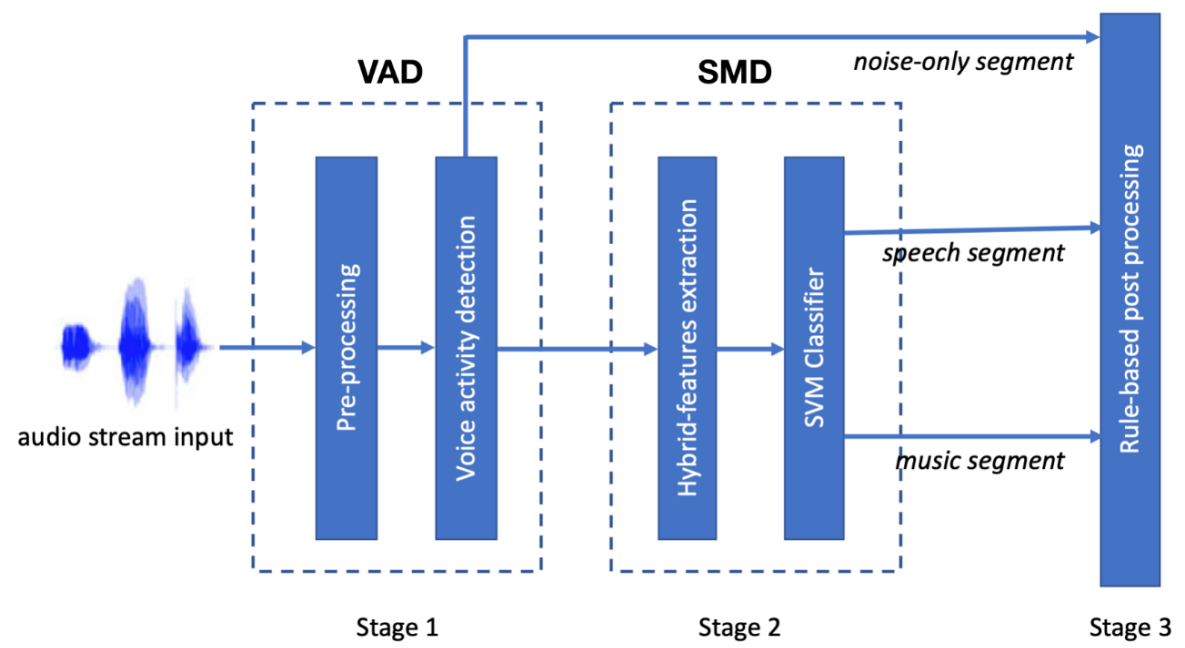

Figure 2. The flowchart for deriving the proposed hierarchical audio content classification with entropy-based voice activity detection (VAD), hybrid-based speech/music discrimination (SMD), and rule-based post-processing. SVM = support vector machine. 


\subsection{Pre-Processing}

In order to emphasize the important higher-frequency elements, the speech signal is first high-pass filtered. The speech frame, $x[n]$, is then divided into several segments. Those segments are chosen as frame size $=256$ samples and 50\% overlapping with the neighboring frame. The Hamming window is applied to each segment after frame partitioning [58].

\subsection{Spectral Entropy-Based Voice Activity Detection (VAD)}

The VAD differs from speech/music discrimination (SMD). VAD discriminates between noise and speech while SMD discriminates between speech and music [1-7].

The conventional VAD algorithms rely on short-time energy or spectral energy as the primary feature parameters with the augmentation of zero-crossing rate, pitch, and duration information [59,60]; yet these features become less reliable in the presence of non-stationary noise and various types of sound artifacts. Extended from previous works [53,61], a spectral entropy-based voice activity detection (VAD) scheme was successfully used for segmenting the noisy signal into noise-only segments and noisy audio segments especially for variable noise-level. Herein, the spectral entropy-based VAD is utilized in the first stage of the ACC system.

In a previous work [61], the band-partitioning spectral entropy (BSE) parameter, $H_{B S E}$, was presented as follows:

$$
H_{B S E}=\sum_{m=1}^{N_{b}} W(m) \cdot P(m) \cdot \log [1 / P(m)]
$$

where $N_{b}$ is the total band size of each frame ( $N_{b}=32$ uniform-bands). $W(m)$ indicates the weight of the $m^{\text {th }}$ band defined as follows:

$$
W(m)=\operatorname{var}\left[\min \left(P_{b}\right) / P_{b}(m-1), P_{b}(m), P_{b}(m+1)\right]
$$

where $\operatorname{var}(\cdot)$ represents the variance. $P_{b}(m)$ represents the probability associated with band energy described as follows:

$$
P_{b}(m)=E_{b}(m) / \sum_{k=1}^{N_{b}} E_{b}(k), \quad 1 \leq m \leq N_{b}
$$

where $E_{b}(m)$ represents the band energy of the $m^{\text {th }}$ band.

Figure 3 shows that the measurement of spectral entropy is robust against changing signal levels even though the amplitude of background noise varies with the environmental state because the spectral entropy depends only on the variation of spectral energy, but not on the amount of spectral energy.
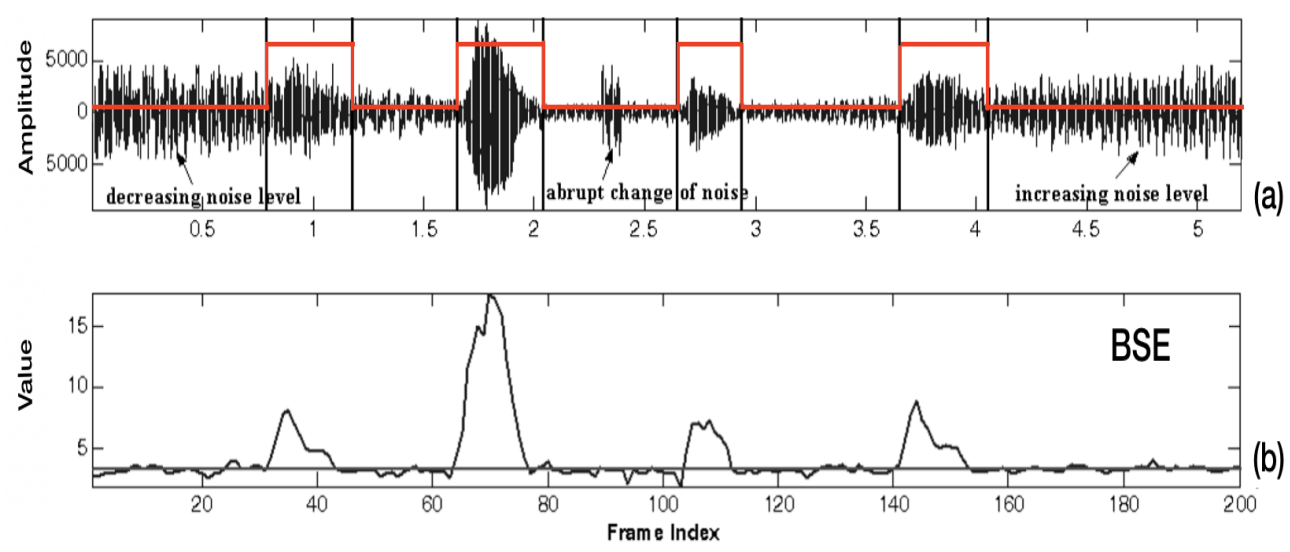

Figure 3. The measurement of spectral entropy is robust against changing signal levels even though the amplitude of background noise varies with the environmental state. (a) The audio signal mixed with variable-noise level. (b) The value of band-partitioning spectral entropy (BSE) parameter over a period of time. 


\section{Hybrid-Based Speech/Music Discrimination (SMD)}

The processing flow of the hybrid-based SMD is shown in Figure 4. The SMD is based on a hybrid feature set, which contains 1D subband energy information (1D-SEI) and 2D texture information (2D-TII) parameters. For noisy segmented audio input, the composed features are extracted from the 1D-PWPT and Bark scale spectrogram image, respectively. The hybrid features include 1D-SEI feature set and 2D-TII feature set. For the feature extraction of 1D-SEI, we used 1D-PWPT (perceptual wavelet packet transform) to get 24 critical subbands. Through the useful subband selection, the correct energy information was used to discriminate the difference between speech and music. In the feature extraction of 2D-TII, gray-scale spectrogram was first generated. Zoning the range from $0 \mathrm{kHz}$ to $4 \mathrm{kHz}$, the local information is enough to characterize speech and music, respectively. Using 2D-PWPT, we can get the 2D textural information. Finally, the hybrid features are then fed into the SVM-based classifier to discriminate their types (speech or music).

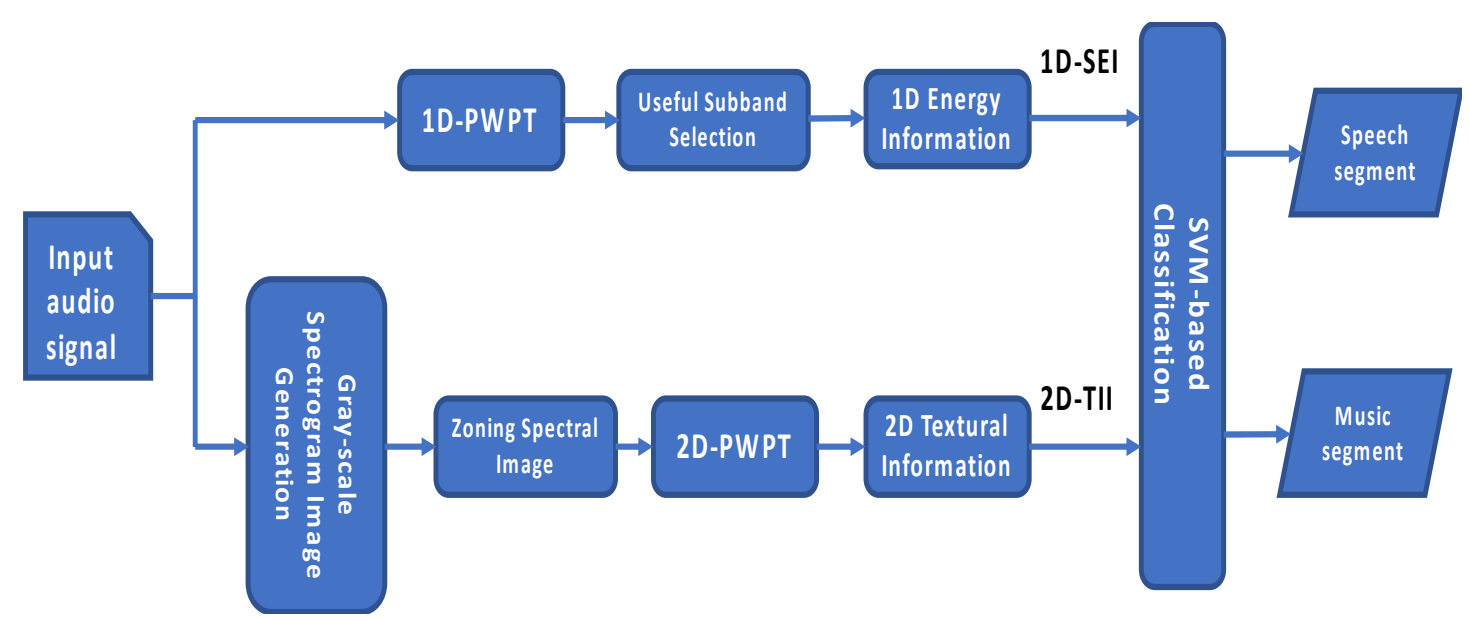

Figure 4. The flowchart of the hybrid-based speech/music discrimination. 1D/2D-PWPT = one/twodimensional perceptual wavelet packet transform.

\subsection{D-PWPT (Perceptual Wavelet Packet Transform)}

In order to mimic the hearing characteristics of human cochlea, the Bark scale, a psychoacoustical scale proposed by Eberhard Zwicker in 1961, was used [62]. It was found that for the auditory quality of a speech signal, an analysis on non-uniform frequency resolution is better than on uniformly spaced frequency resolution [63]. In fact, the selection of the "optimal" decomposition is a classical problem in order to suppress audible noise and eliminate audible artefacts. According to the Bark scale rules, the 1D-perceptual wavelet packet transform (PWPT) implemented with an efficient five-stage tree structure is utilized to split 24 critical subbands for input speech signal. For each stage, the high-pass filter and low-pass filter are implemented with the Daubechies family wavelet, where the symbol $\downarrow 2$ denotes an operator of down-sampling by 2 [53]. In Table 1, we see that the Bark scale-based wavelet decomposition lets every frequency band limit become more and more linear when frequencies are below $500 \mathrm{~Hz}$; this scale is more or less equal to a logarithmic frequency axis when above about $500 \mathrm{~Hz}$.

Table 1. Frequency bands limits, in $\mathrm{Hz}$, for Bark scale vs. wavelet scale.

\begin{tabular}{cccccc}
\hline \multirow{2}{*}{ Band Index } & \multicolumn{2}{c}{ Bark Scale } & \multicolumn{3}{c}{ Wavelet Scale } \\
\cline { 2 - 6 } & Bandlimit & Bandwidth & Bandlimit & Bandwidth & Transform Stage \\
\hline 1 & {$[0-100]$} & 100 & {$[0-125]$} & 125 & $\# 5$ \\
\hline 2 & {$[100-200]$} & 100 & {$[125-250]$} & 125 & $\# 5$ \\
\hline 3 & {$[125-250]$} & 100 & {$[250-375]$} & 125 & $\# 5$ \\
\hline
\end{tabular}


Table 1. Cont.

\begin{tabular}{|c|c|c|c|c|c|}
\hline \multirow{2}{*}{ Band Index } & \multicolumn{2}{|c|}{ Bark Scale } & \multicolumn{3}{|c|}{ Wavelet Scale } \\
\hline & Bandlimit & Bandwidth & Bandlimit & Bandwidth & Transform Stage \\
\hline 4 & [300-400] & 100 & [375-500] & 125 & $\# 5$ \\
\hline 5 & [400-510] & 110 & [500-625] & 125 & $\# 5$ \\
\hline 6 & [510-630] & 120 & [625-750] & 125 & $\# 5$ \\
\hline 7 & [630-770] & 140 & [750-875] & 125 & $\# 5$ \\
\hline 8 & [750-875] & 150 & [875-1000] & 125 & $\# 5$ \\
\hline 9 & [920-1080] & 160 & [1000-1250] & 125 & $\# 4$ \\
\hline 10 & [1080-1270] & 190 & [1250-1500] & 250 & $\# 4$ \\
\hline 11 & [1270-1480] & 210 & [1500-1750] & 250 & $\# 4$ \\
\hline 12 & [1480-1720] & 240 & [1750-2000] & 250 & $\# 4$ \\
\hline 13 & [1720-2000] & 280 & [2000-2250] & 250 & $\# 4$ \\
\hline 14 & [2000-2320] & 320 & [2250-2500] & 250 & $\# 4$ \\
\hline 15 & [2320-2700] & 380 & [2500-3000] & 500 & $\# 4$ \\
\hline 16 & [2700-3150] & 450 & [3000-3500] & 500 & $\# 4$ \\
\hline 17 & [3150-3700] & 550 & [3500-4000] & 500 & $\# 4$ \\
\hline 18 & [3700-4400] & 700 & [4000-5000] & 1000 & $\# 4$ \\
\hline 19 & [4400-5300] & 900 & [5000-6000] & 1000 & $\# 3$ \\
\hline 20 & [5300-6400] & 1100 & [6000-7000] & 1000 & $\# 3$ \\
\hline 21 & [6400-7700] & 1300 & [7000-8000] & 1000 & $\# 3$ \\
\hline 22 & [7700-9500] & 1800 & [8000-10,000] & 2000 & $\# 2$ \\
\hline 23 & {$[9500-12,000]$} & 2500 & {$[10,000-12,000]$} & 2000 & $\# 2$ \\
\hline 24 & {$[12,000-15,500]$} & 3500 & {$[12,000-16,000]$} & 4000 & $\# 2$ \\
\hline
\end{tabular}

\subsection{Optimal Subband Selection for Useful Information}

In previous works [64], an extraction of selecting useful frequency subbands was proposed to suppress the noise effect on the ACC system, especially at a poor SNR (signal-to-noise ratio). The process of pure energy on the useful frequency is shown below.

During the initialization period, the noisy signal was assumed to be noise-only, and the noise spectrum was estimated by averaging the initial 10 frames. To recursively estimate the noise power spectrum, the subband noise power, $\bar{N}(\zeta, m)$, was adaptively estimated by smoothing filtering.

For the $m^{\text {th }}$ frame, the spectral energy of the $\zeta^{\text {th }}$ subband is evaluated by the sum of squares:

$$
E(\zeta, m)=\sum_{\omega_{\zeta, l}}^{\omega_{\zeta, h}}|w(\omega, m)|^{2}
$$

where $w(\omega, m)$ means the $\omega^{\text {th }}$ wavelet coefficient. $\omega_{\zeta, l}$ and $\omega_{\zeta, h}$ denote the lower boundaries and the upper boundaries of the $\zeta^{\text {th }}$ subband, respectively.

The $\zeta^{\text {th }}$ frequency subbands energy of pure speech signal of the $m^{\text {th }}$ frame $\widetilde{E}(\zeta, m)$ is estimated:

$$
\widetilde{E}(\zeta, m)=E(\zeta, m)-\widetilde{N}(\zeta, m)
$$

where $N(\zeta, m)$ is the noise power of the $\zeta^{\text {th }}$ frequency subband. 
According to Wu et al. [65], subbands with a higher energy $\widetilde{E}(\zeta, m)$ can stand for a greater amount of pure speech information. So, the frequency subband should be sorted according to its value of $\widetilde{E}(\zeta, m)$.

That is,

$$
E\left(I_{1}, m\right) \geq E\left(I_{2}, m\right) \geq \cdots \geq E\left(I_{N_{u b}(m)}, m\right), I \in\left[I_{1}, I_{2}, I_{3}, \cdots, I_{N_{u b}(m)}\right]
$$

where $I_{i}$ is the index of the frequency subband with the $i^{t h}$ max energy. $N_{u b}(m)$ denotes the number of useful subbands on the $m^{\text {th }}$ frame. $I \in\left[I_{1}, I_{2}, I_{3}, \cdots, I_{N_{u b}(m)}\right]$.

In fact, the relation between the number of useful frequency subbands, $N_{u b}(m)$, and the posterior SNR, SNR $(m)$, has a negative-correlation, as shown in Figure 5.

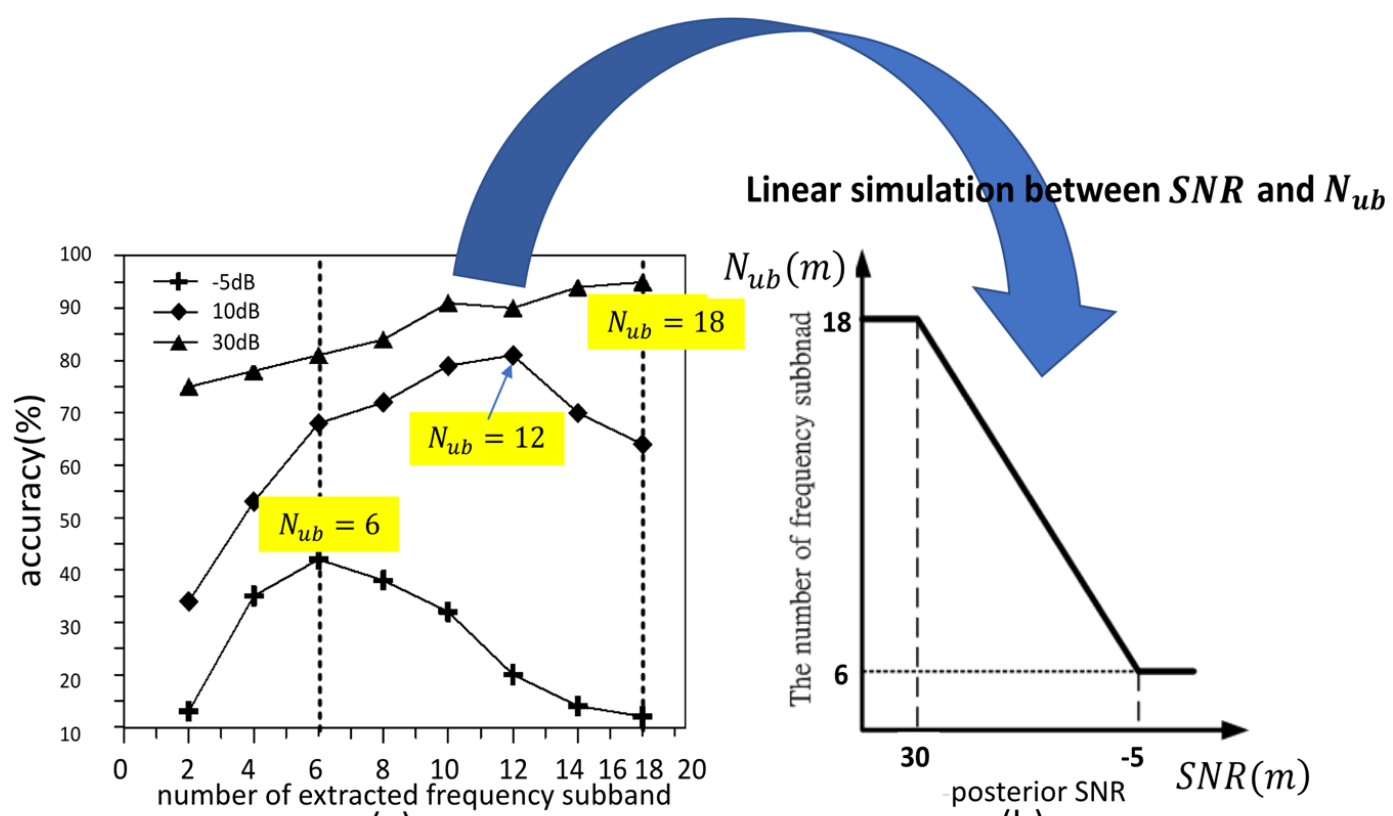

(a)

(b)

Figure 5. The relation between the number of useful frequency subbands, $N_{u b}(m)$, and the posterior signal-to-noise ratio (SNR), $S N R(m)$. (a) The results of correct detection accuracy with different frequency subbands at $-5 \mathrm{~dB}, 10 \mathrm{~dB}$, and $30 \mathrm{~dB}$. (b) A linear function of the relationship between $N_{u b}(m)$ and $\operatorname{SNR}(m)$.

We see that the number of useful frequency subbands increases with the increase of SNR in Figure 5a. When $S N R(m)=-5, S N R(m)=10$, and $S N R(m)=30 \mathrm{~dB}$, the highest accuracy of VAD appears as $N_{u b}(m)=6, N_{u b}(m)=12$, and $N_{u b}(m)=18$, respectively. In order to simulate the relationship between $N_{u b}(m)$ and $S N R(m)$, a linear function is in the boundary between $-5 \mathrm{~dB}$ and $30 \mathrm{~dB}$, while the duration between $N_{u b}(m)=6$ to $N_{u b}(m)=18$ is shown in Figure $5 \mathrm{~b}$ :

$$
N_{u b}(m)=\left[\begin{array}{cc}
6, & S N R(m)<-5 \mathrm{~dB} \\
\left.(18-6) \times \frac{(S N R(m)-(-5))}{30-(-5)}+6\right], & -5 \mathrm{~dB} \leq S N R(m) \leq 30 \mathrm{~dB} \\
18, & S N R(m)>30 \mathrm{~dB}
\end{array}\right.
$$

where [.] is the round off operator and $S N R(m)$ denotes a frame-based posterior $S N R$ for the $m^{\text {th }}$ frame. $\operatorname{SNR}(m)$ is dependent on the summation of subband-based posterior SNR $\operatorname{snr}(\zeta, m)$ on the $\zeta^{\text {th }}$ useful subband, defined as:

$$
\operatorname{SNR}(m)=10 \times \log _{10} \sum_{\zeta \in N_{u b}} \operatorname{snr}(\zeta, m),
$$

where $\operatorname{snr}(\zeta, m)=|E(\zeta, m)|^{2} / \widetilde{N}(\zeta, m)$. 
Figure 6 clearly illustrates the example of extracting useful subbands under a different posterior SNR. We see that the pure subband energy is rearranged after sorting processing among all 24 subbands. Originally, the first subband index $\zeta$ is 1 , but the updated first index $\zeta$ is 3 when sorting the energy. Consequently, the useful subband index and number are extracted according to the value of the posterior SNR.
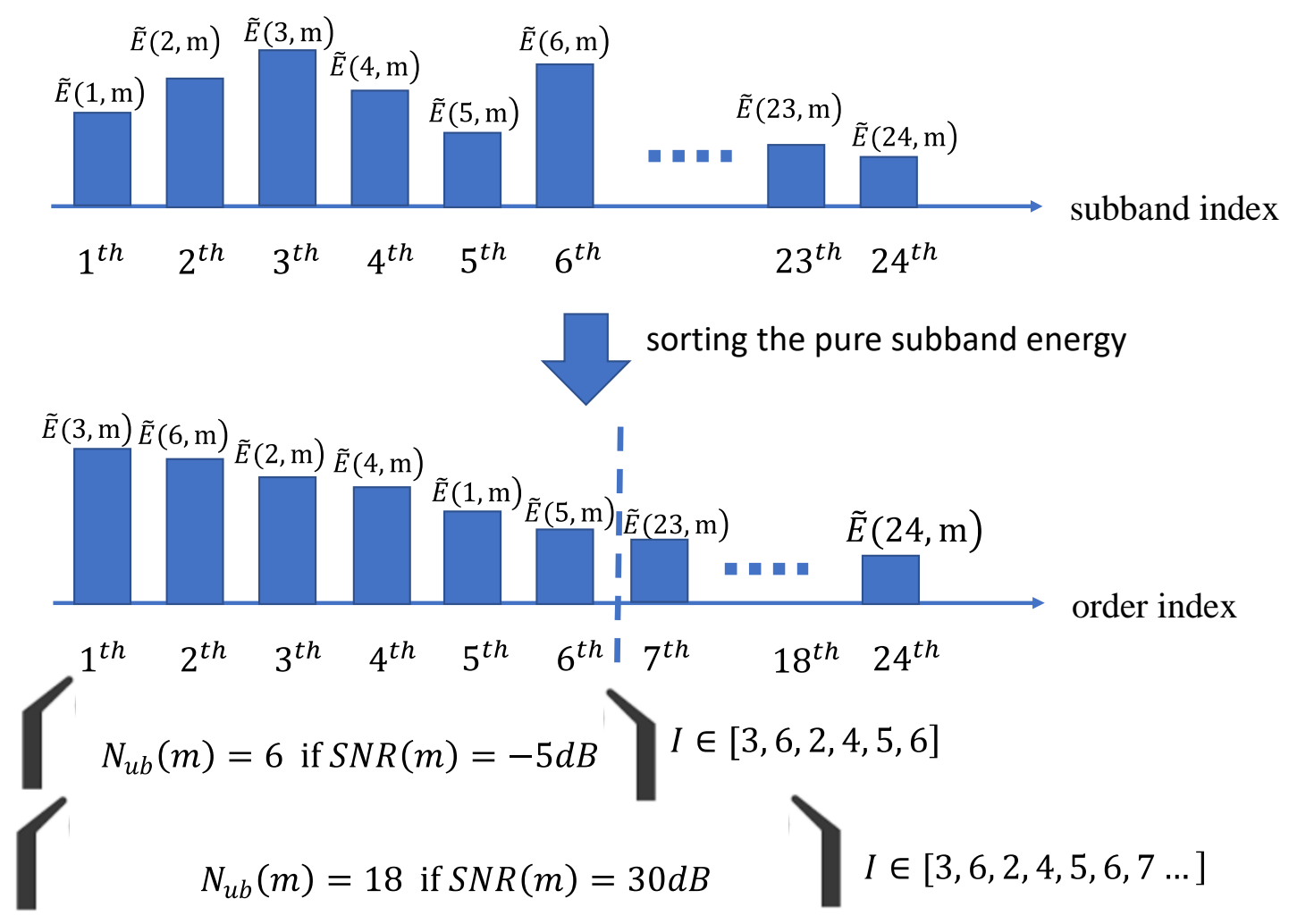

Figure 6. Example for extracting the useful subband index and number according to the value of the posterior SNR.

\subsection{The 1D Subband Energy Informations (1D-SEIs)}

It is well-known that the distribution of energy on each frequency band is a very relevant acoustic cue. After selecting a useful subband, the wavelet energy was calculated from 1D-PWPT to form a 1D subband energy informations (1D-SEIs): the average of subband energy (ASE), the standard deviation of subband energy (SDSE), and Teager energy. So, the 1D-SEIs derived from three parameters are investigated below:

- $\quad$ The average of subband energy (ASE)

$$
E^{a v g}(m)=\sum_{1}^{N_{u b}(m)} \widetilde{E}(\zeta, m) / N_{u b}(m), \zeta \in\left[I_{1}, I_{2}, I_{3}, \cdots, I_{N_{u b}(m)}\right] .
$$

- $\quad$ The standard deviation of subband energy (SDSE)

$$
E^{s d}(m)=1+\sqrt{\frac{1}{24} \sum_{\zeta=1}^{24}\left(\widetilde{E}(\zeta, m)-E^{a v g}(m)\right)^{2}}
$$

We see that the speech's energy exists in a lower frequency band mainly and the music's energy is in a wide range of the frequency band. 
- $\quad$ Teager energy

$$
E^{\operatorname{teg}}(m)=\widetilde{E}(\zeta, m)^{2}-\widetilde{E}(\zeta, m-1) \times \widetilde{E}(\zeta, m+1)
$$

The discrete Teager energy operator (TEO), introduced by Kaiser [66], allows modulation energy tracking and gives a better representation of the formant information in the feature vector. So, we can also successfully use the characteristic to discriminate speech from music.

\subsection{Gray-Scale Spectrogram Image Generation}

In this subsection, a novel feature extraction is derived from the gray-scale spectrogram images. As mentioned above, we see the difference between speech and music while relying on the virtual representation of audio data by spectrogram. In fact, the gray-scale spectrogram images are regarded as a time-frequency-intensity representation. Since the human perception of sound is logarithmic, the log-spectrogram is defined as:

$$
S_{\log }(k, t)=\log (|X(k, t)|) .
$$

The time-frequency-intensity representation is normalized into a grayscale normalized image, within the range of 0 to 1 :

$$
R_{\text {SpecImg }}(k, t)=\left(S_{\text {log }}(k, t)-S_{\text {min }}\right) /\left(S_{\max }-S_{\text {min }}\right) .
$$

\subsection{The Zoning for Spectrogram Image}

To achieve good results for SMD, the zoning method for spectrogram image was applied [67]. In fact, the textural image information between speech signals and music data is different [68]. It was found that the music audio data consist of a few silent intervals, and have continuous energy peaks for a short time and fewer frequency variations, while the speech audio data consist of many silent intervals and most of the energy is located at the lower frequencies [69]. Accordingly, the spectrogram image from $0 \mathrm{kHz}$ to $4 \mathrm{kHz}$ is separated to extract textural features as local features by the zoning method. The feature extraction for the 2D textural image information (2D-TII) is discussed in the next subsection.

\subsection{The 2D Textural Image Information (2D-TII)}

In fact, the differences on the sound spectrogram between music and speech are significant. In music, the spectrum's peak tends to change relatively slowly even though the music is played with various tempos. On the contrary, in speech, sound events often have shorter durations but with more distinctive time-frequency representations. For the above reason, the 2D-TII features can be successfully derived from the audio spectrogram image through Laws' masks based on the principle of texture energy measurement [54] to find the difference between speech and music. It is known that Laws' masks are well described for texture energy variation in image processing, and the masks consist of five masks derived from one-dimensional vectors, such as edge $E_{5}$, level $L_{5}$, spot $S_{5}$, ripple $R_{5}$, and wave $W_{5}$ expressed as Equations (14)-(18):

$$
\begin{aligned}
E_{5} & =\text { Edge detection }:\left[\begin{array}{llllll}
-1 & -2 & 0 & 2 & 1
\end{array}\right] \\
L_{5} & =\text { Level detection }:\left[\begin{array}{llllll}
1 & 4 & 6 & 4 & 1
\end{array}\right] \\
S_{5} & =\text { Spot detection : }\left[\begin{array}{llllll}
-1 & 0 & 2 & 0 & -1
\end{array}\right] \\
R_{5} & =\text { Ripple detection : }\left[\begin{array}{llllll}
1 & -4 & 6 & -4 & 1
\end{array}\right] \\
W_{5} & =\text { Wave detection : }\left[\begin{array}{llllll}
-1 & 2 & 0 & -2 & 1
\end{array}\right]
\end{aligned}
$$


The two-dimensional filters of the size $5 \times 5$ were generated by convoluting any vertical one-dimensional vector with a horizontal one. Finally, the 25 combinations of two-dimensional masks are determined [70].

First, we convoluted the image with each two-dimensional mask to extract texture information from an image $I_{(i, j)}$ of size $(M \times N)$. For example, if we used $E_{5} E_{5}$ to filter the image $I_{(i, j)}$, the result was a texture image, $T I_{E_{5} E_{5}}$, as seen in Equation (19).

$$
T I_{E_{5} E_{5}}=I_{i, j} \otimes E_{5} E_{5}
$$

All the two-dimensional masks, except $L_{5} L_{5}$, had a zero mean. According to Laws, texture image $T I_{L_{5} L_{5}}$ was used to normalize the contrast of all the texture images $T I_{(i, j)}$, as seen in Equation (20).

$$
\operatorname{Normalize}\left(T I_{\text {mask }}\right)=T I_{\text {mask }} / T I_{L_{5} L_{5}} \text {. }
$$

Next, the outputs (TI) from Laws' masks were passed to "texture energy measurement" (TEM) filters. We calculate the non-linear interval by processing TI normalized and yield through "Texture Energy Measurements, (TEM)" filter. This consisted of a moving non-linear window average of absolute values, as seen in Equation (21).

$$
\operatorname{TEM}_{i j}=\sum_{u=-7}^{u=7} \sum_{v=-7}^{v=7}\left[\operatorname{Normalize}\left(T I_{i+u, j+v}\right)\right] .
$$

Since not all mask energy is used as the input basis of texture energy, we take out unchangeable $T R$ values before and after rotation to obtain a valid TEM. The $T R$ derived from $T E M$ is represented in Equation (22).

$$
T R_{E 5 L 5}=\left(T E M_{E 5 L 5}+T E M_{L 5 E 5}\right) / 2
$$

After Equation (22), the results of the three texture feature values: mean, standard deviance (SD), and entropy are extracted via Equations (23)-(25) to exploit the variation of texture information.

$$
\begin{gathered}
\text { Mean }=\sum_{i=0}^{M} \sum_{j=0}^{N} T R_{i j} /(M \times N), \\
S D=\sqrt{\sum_{i=0}^{M} \sum_{j=0}^{N}\left(T R_{i j}-M e a n\right)^{2} /(M \times N),} \\
\text { Entropy }=\sum_{i=0}^{M} \sum_{j=0}^{N} T R_{i j}{ }^{2} /(M \times N) .
\end{gathered}
$$

Each equation produces feature vectors with 14-dimensional size. Finally, a total of three feature vectors with 42-dimensional sizes are used as the input data for training the SVM classifier.

\subsection{From $2 D-P W P T$ to $2 D-T I I$}

To perform texture analysis on multi-resolution, 2D-PWPT is utilized into an audio spectrogram image, which ranges from 0 to $4 \mathrm{KHz}$. Figure 7 shows an audio spectrogram image decomposition. In Figure 7, these subbands are first obtained using one-level wavelet decomposition. These subbands are labeled as LH1, HL1, and HH1 and represent the detail images, while the sub-band labeled as LL1 is regarded as the approximation image. The detail images represent the finest scale wavelet coefficients. Conversely, the approximation image corresponds to coarse level coefficients. The sub-band LL1 alone is further decomposed and critically sampled in order to obtain the next coarse level of wavelet coefficients. So, this results in two-level wavelet decomposition. Similarly, LL2 is used to obtain further decomposition. Lastly, the spectrogram image of LL2 is only convoluted by the two-dimensional Laws' mask to determine the 2D-TII. Compared to the original image size of the spectrogram within 0 to $4 \mathrm{kHz}$, the LL2 is de-sized. Thus, we can decrease the computing time and get good information derived from LL2 sub-image that is better than the original image. 


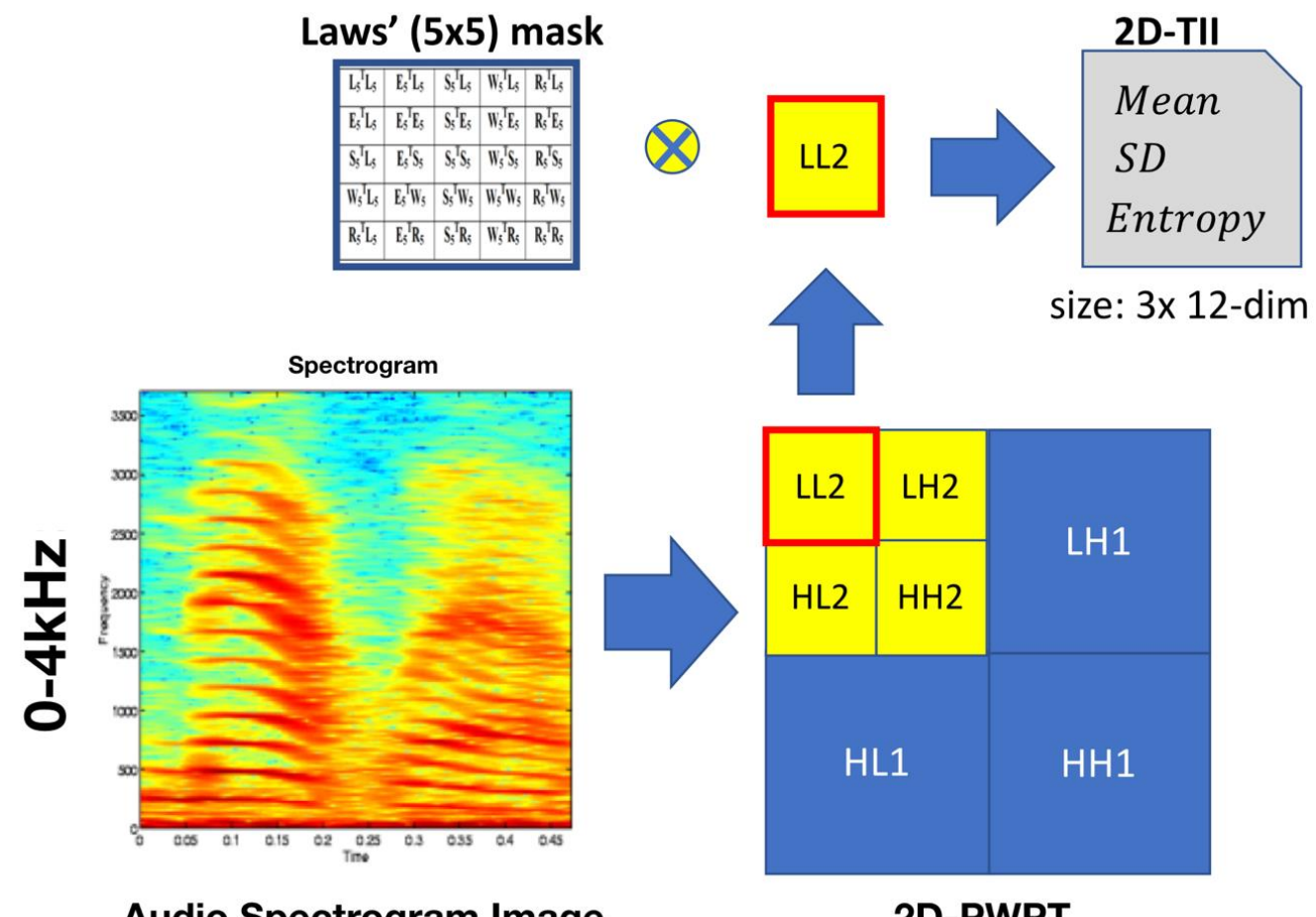

Figure 7. The block-diagram of the two-dimensional textural image information (2D-TII) derived from LL2 sub-image.

\subsection{SVM-Based Classification}

Support vector machine (SVM) is well-known effective bi-classification [71-73]. In actuality, the SVM is better than other conventional classifiers in terms of classification accuracy, computational time, and stability. In this subsection, the hybrid feature set including 1D-subband energy information and 2D-texture information, $F^{h y b}=\left[1 \mathrm{D} \_S E I, 2 \mathrm{D} \_\mathrm{TII}\right]$, are imported into a discriminative classifier of the SVM to classify either the speech segment or music segment. Suppose a set $S=\left\{\left(x_{1}, y_{1}\right), \ldots,\left(x_{N}, y_{N}\right)\right\}_{\text {of }} R^{n}$ is the training set, where $x_{i}$ is the input signal vector, $y_{i}$ is the class label for speech or audio, $y_{i} \in\{-1,1\}$, and $R^{n}$ denotes $n$-dimensional space.

To find the optimal hyper-plane, the support vectors of the dataset maximize the margin, which is the distance between the hyper-plane and support vectors as follows:

$$
\begin{gathered}
\min \frac{1}{2}\|w\|^{2} \\
\text { s.t. } y_{i}\left(w^{T} x_{i}+b\right) \geq 1
\end{gathered}
$$

The solution to the optimization problem of SVM is given by the Lagrange function as follows:

$$
L(\alpha)=\sum_{i=1}^{N} \alpha_{i}-\frac{1}{2} \sum_{i=1}^{N} \sum_{j=1}^{N} \alpha_{i} \alpha_{j} y_{i} y_{j} K\left(x_{i}, x_{j}\right)
$$

with constraint $\sum_{i=1}^{N} \alpha_{i} y_{i}=0$ and $0 \leq \alpha_{i} \leq C$, where $C$ is upper bound of the Lagrange multipliers $\alpha_{i}$ and the constant $C \in[0,1]$.

As for the kernel function, we consider ERBF and Gaussian function as shown below:

$$
\begin{gathered}
K_{\mathrm{ERBF}}(\mathrm{x}, \mathrm{y})=\exp \left(-\gamma|x-y| / 2 \sigma^{2}\right), \\
K_{\mathrm{Gaussian}}(\mathrm{x}, \mathrm{y})=\exp \left(-\gamma|x-y|^{2} / 2 \sigma^{2}\right),
\end{gathered}
$$


where $\sigma^{2}$ is the variance. $\gamma$ is the additional control parameter.

Potentially, the ERBF function is usually used as the kernel function and vastly improves the results [74]. Therefore, the SVM which adopts ERBF as a kernel function will be compared to other classification.

\section{The Rule-Based Post-Processing}

The purpose of the post-processing step is to reduce possible errors of segmentation and classification. The errors of segmentation may even be occurred due to abrupt changes in noise level. Here are some examples of rule-based schemes used in the post-processing step: if a "music" segment appears separately in a series of speech segments, it merges into that speech segment; if a "speech" segment appears separately in a series of music segments, it merges into that music segment; if a "music" segment appears in only two frames or is smaller than two frames, it merges into speech segments. The kernel of a rule-based engine is regarded as a set of IF-THEN rules. The formulations of a rule-based engine where speech is ' $\mathrm{S}$ ", music is " $\mathrm{M}$ ', noise/silence/other is " $\mathrm{N}$ ', and " _" is represented as any audio type except for noise can be shown below:

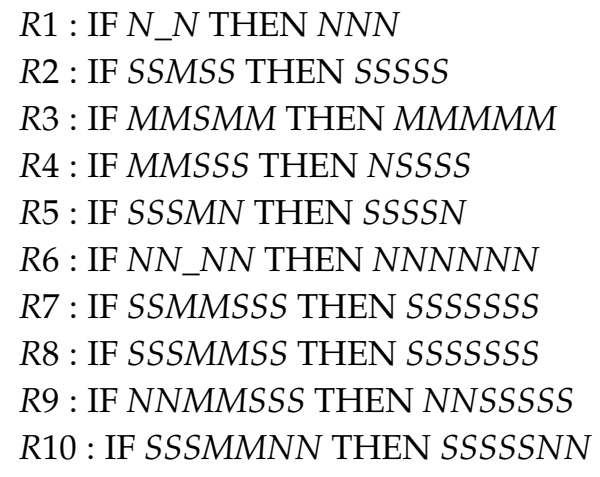

According to R1 to R10 from Equation (30), the procedure of a rule-based post-processing is fulfilled by the smoothing task as shown in Figure 8. Observing the figure, the hybrid features from SVM and VAD are regarded as input. After a complete loop over all the rules, the loop is repeated, until the segmentation remains unchanged.

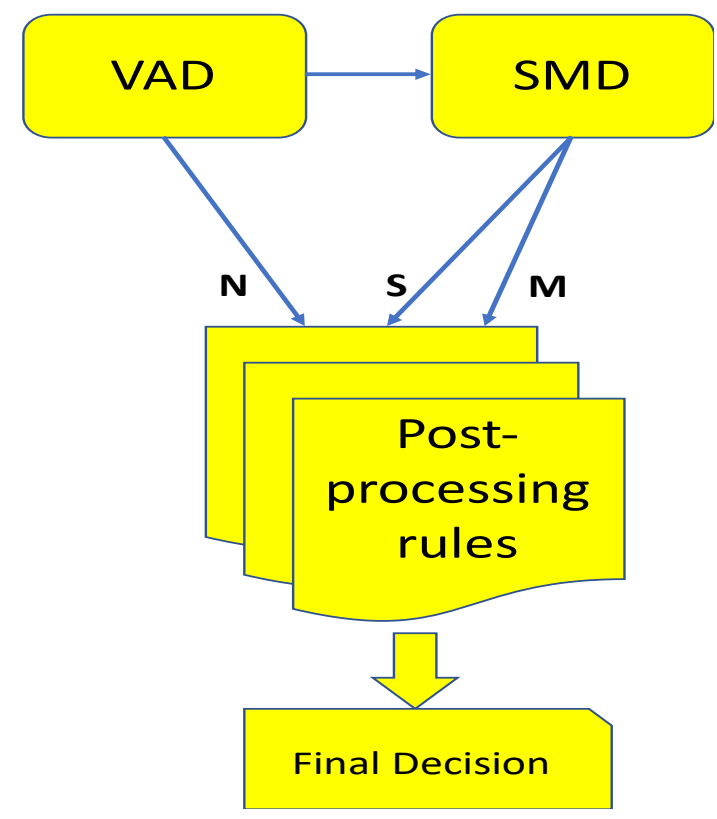

Figure 8. The block diagram of rule-based post-processing. 


\section{Experimental Results}

\subsection{Database Description}

To evaluate the proposed algorithm, the database consisted of three different subsets shown in Table 2. The first one is the well-known Music-Speech GTZAN library [75], which includes 120 tracks, each lasting $30 \mathrm{~s}$ and containing 60 examples of each class (music/speech). The second one is artificial audio data, which are artificially created by concatenating silence, speech, or music segments. The last one is real broadcasting recordings, which were collected from BBC radio, NHK, and TTV news. Subsets \#2 and \#3 were collected and organized from artificial audio data and real broadcasting recordings. The speech data come from news programs on the radio and TV stations, talks, as well as dialogs in movies, and the languages involve English, Chinese, and Japanese. In addition, the music consists of instrumental music and songs obtained from music CDs covering classic, pop, folk, and rock. The audio data stream was sampled in 16-bit with $8 \mathrm{kHz}$. In addition, many publicly available audio datasets including LVLib-SMO, FMA-small, and RWC Music Database are also introduced in Subsets \#4, \#5, and \#6.

Table 2. The evaluation dataset.

\begin{tabular}{|c|c|c|c|}
\hline$\#$ & Duration & Type & Subset \\
\hline 1 & $3840 \mathrm{~s}$ & Music, speech & GTZAN Music-Speech [75] \\
\hline 2 & $12 \mathrm{~min}$ & Music, speech, silence & Artificial Audio Data \\
\hline 3 & $14 \mathrm{~min}$ & Music, Speech, silence, other & Real Radio Recordings \\
\hline 4 & $7 \mathrm{~h} 37 \mathrm{~min}$ & Music, speech, other & LVLib-SMO [12] \\
\hline 5 & 8000 tracks of $30 \mathrm{~s}$ & $\begin{array}{c}\text { Top } 8 \text { genres, balanced with } 1000 \text { clips per } \\
\text { genre, } 1 \text { root genre per clip }\end{array}$ & FMA-small dataset [76] \\
\hline 6 & $91.6 \mathrm{~h}$ & Popular, classical, and jazz music databases & RWC Music Database [77] \\
\hline
\end{tabular}

\subsubsection{Artificial Audio Data}

Three test files were artificially created by concatenating silence, speech, or music segments. The speech signals spoken by a variety of both male and female speakers were taken from 12 to $15 \mathrm{~min}$. The composition of the data set is shown as follows:

- Arti Num. 1: This is 15 min audio stream with alternate speech, music, and silence segments of equal ( $30 \mathrm{~s}$ ) duration. This data set includes $12.54 \%$ of silence, $42.78 \%$ of speech, and $44.68 \%$ of music.

- Arti Num. 2: This data set also consists of 15 min audio stream comprising mainly of music data. In this case, 20 segments of music data are interleaved with shorter segments of speech. Therefore, this data set is composed of $9.36 \%$ of silence, $22.57 \%$ of speech, and $68.07 \%$ of music.

- Arti Num. 3: This data set also consists of 15 min audio stream comprising mainly of speech data. In this case, $20 \mathrm{~s}$ segments of music data are interleaved with shorter segments of speech. Therefore, this data set is composed of $11.58 \%$ of silence, $64.38 \%$ of speech, and $24.04 \%$ of music.

The results of three artificial files with different combinations of the sounds are shown in Table 3.

Table 3. The percentage of distribution between speech, music, and noise.

\begin{tabular}{cccc}
\hline Arti Num. & Silence & Speech & Music \\
\hline 1 & $12.54 \%$ & $42.78 \%$ & $44.68 \%$ \\
2 & $9.36 \%$ & $22.57 \%$ & $68.07 \%$ \\
3 & $11.58 \%$ & $64.38 \%$ & $24.04 \%$ \\
\hline
\end{tabular}




\subsubsection{Real Radio Recordings}

The real TV news and real movie clips were recorded from BBC radio, NHK, and TTV news in order to evaluate the results of the proposed algorithm under realistic noisy environments. The durations of two real recording files range from 10 to $15 \mathrm{~min}$ where the length of the silence segment varies from $1 \mathrm{~s}$ to $3 \mathrm{~s}$ and the length of speech or music segments vary from $3 \mathrm{~s}$ to $10 \mathrm{~s}$. To evaluate whether the proposed ACC algorithm is valid for a realistic environment, Table 4 shows the real TV news and real movie clips selected as Real \#01 and Real \#02, respectively.

Table 4. The composition of real radio recordings.

\begin{tabular}{cccc}
\hline Real Num. & Length & Type & Source \\
\hline 1 & $12 \mathrm{~min}$ & TV news clip & NHK and TTV news \\
\hline 2 & $14 \mathrm{~min}$ & Movie clip & BBC radio \\
\hline
\end{tabular}

\subsection{Evaluation Results of Entropy-Based VAD}

In the first evaluation, the experimental results of the entropy-based VAD is presented. The goal of VAD segmentation is to divide the audio signal into a voice segment (including speech and music) and non-voiced segment (including noise and background silence). Figure 9 shows segmentation performance of the entropy-based VAD proposed in our earlier work [53,61] against any variable noise-level conditions when comparing to the conventional method. It is found that the entropy-based parameter is related only to the variation of spectral energy but not to the amount of spectral energy, so the entropy-based algorithm outperforms the energy-based algorithm, especially in changing the level of noise.
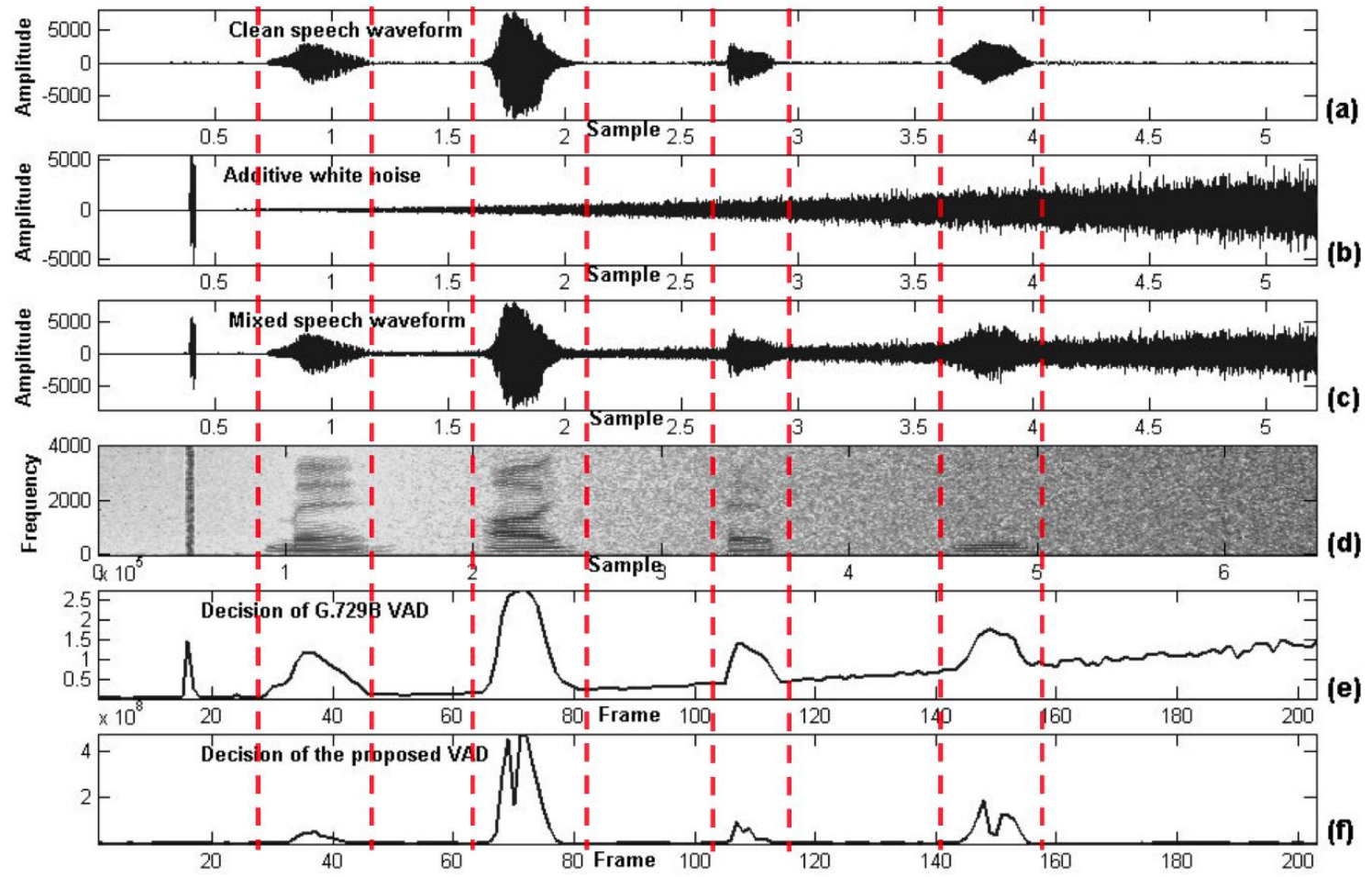

Figure 9. Comparison between the proposed VAD and G.729B. (a) Waveform of a clean speech 'May I help you?'. (b) Waveform of an additive factory noise. (c) Waveform of a mixed speech signal. (d) Spectrogram of a mixed speech signal. (e) Result of G.729B VAD. (f) Result of the proposed VAD. 
To further evaluate the performance of the proposed VAD method, the following metrics were considered:

$$
\begin{gathered}
\text { Accuracy }[\%]=\frac{T P+T N}{T P+F P+T N+F N} \\
\text { Precision }[\%]=\frac{T P}{T P+F P} \\
\text { Recall }[\%]=\frac{T P}{T P+F N} \\
\text { F1-score }[\%]=\frac{2}{\frac{1}{\text { Precision }}+\frac{1}{\text { Recall }}}
\end{gathered}
$$

where $T P, F P, T N$, and $F N$ represent true positive, false positive, true negative, and false negative rates, respectively. Accuracy[\%] denotes the ratio correctly classified as voiced and non-voiced segments. Precision $[\%]$ indicates the ratio correctly classified as voiced segments when predicting a voiced segment. Recall $[\%]$ means the true positive rate when predicting a voiced segment and non-voiced segment. F1-score $[\%]$ is derived by calculation of the harmonic mean value between Precision $[\%]$ and Recall $[\%]$. To examine the effectiveness of the entropy-based VAD, we compared it with other existing VAD methods. Table 5 shows the performance of the proposed VAD in terms of Acc[\%], Pre[\%], Rec[\%], and F1[\%] averaged over the SNR range from $-5 \mathrm{~dB}$ to $30 \mathrm{~dB}$ testing on artificial audio data mixed background noise. In terms of accuracy, we found that the proposed entropy-based algorithm obtains the best Acc $[\%]$ with $90.04 \%$. However, accuracy alone is not enough, and it is usually supplemented by Precision $[\%]$, Recall $[\%]$, and F1-score $[\%]$. The results clearly show that the proposed entropy-based VAD is almost superior to other compared VADs.

Table 5. Performance between various VADs under noise conditions.

\begin{tabular}{ccccc}
\hline Method & Acc [\%] & Pre [\%] & Rec [\%] & F1 [\%] \\
\hline Sohn [78] & 80.66 & 85.58 & 85.92 & 85.75 \\
\hline Ramirez [79] & 89.01 & 88.81 & 91.22 & 90.01 \\
\hline G.729B [80] & 56.69 & 78.31 & 85.33 & 81.67 \\
\hline AMR[81] & 76.52 & 70.33 & 95.86 & 81.14 \\
\hline Tahmasbi [82] & 81.01 & 85.76 & 85.33 & 85.55 \\
\hline Proposed & $\mathbf{9 0 . 0 4}$ & $\mathbf{9 2 . 1 4}$ & $\mathbf{9 2 . 1 2}$ & $\mathbf{9 2 . 1 3}$ \\
\hline
\end{tabular}

\subsection{The Evaluation of Hybrid-Based SMD}

In actually, the Mel-frequency cepstral coefficient (MFCC), zero crossing (ZC), and spectral centroid (SC) are usually used as important features which are applied in the speech/music discrimination systems. In order to present the justification of the proposed hybrid features for speech/music discrimination, a comparison with other commonly used features is tested on data subsets \#1, \#2, and \#3 and shown in Table 6. We see that the proposed hybrid feature set is superior to several well-known feature sets in terms of overall accuracy. The classification accuracy with $96.56 \%$ is the highest value while using a hybrid feature set.

In order to evaluate the performance of hybrid-based speech/music discrimination systems using a SVM classifier with ERBF kernel, the three different statistics are used below:

The percentage of true speech segments identified as speech,

$$
\text { Speech }(\%)=\frac{\text { Correctly Specified Speech Segments }}{\text { Total Speech Segments }}
$$


The percentage of true music segments identified as music,

$$
\operatorname{Music}(\%)=\frac{\text { Correctly Specified Music Segments }}{\text { Total Music Segments }}
$$

The average percentage of correctly identified

$$
\text { Average }(\%)=\frac{\text { Correctly Specified Segments }}{\text { Total Segments }}
$$

Table 7 shows the performance of hybrid-based SMD approach compared to various classifiers on GTZAN dataset. We can see from the results that the SVM classifier with ERBF kernel implies a better performance of the speech/music discriminator. The proposed hybrid-based approach provides the accuracy in Music as $90.41 \%$ due to that it gives rise to an important decrease of the MSE errors. On the contrary, the proposed hybrid-based approach achieves a highest average accuracy rate of $91.33 \%$ (the accuracy in Speech as $92.26 \%$ and in Music as $90.41 \%$ ) among other classifiers.

Table 6. The classification accuracy under different feature sets.

\begin{tabular}{cc}
\hline Feature Set & The Overall Accuracy (\%) \\
\hline Mel-frequency cepstral coefficient (MFCC) & 88.79 \\
\hline Spectral centroid (SC) & 91.67 \\
\hline Zero crossing (ZC) & 76.83 \\
\hline MFCC + SC + ZC & 92.58 \\
\hline 1D-SEI & 90.51 \\
\hline 2D-TII & 94.35 \\
\hline Hybrid feature set: 1D-SEI + 2D-TII & $\mathbf{9 6 . 5 6}$ \\
\hline
\end{tabular}

Table 7. Performance of various classifiers with hybrid features on GTZAN dataset.

\begin{tabular}{cccc}
\hline Classifiers & Speech (\%) & Music (\%) & Average (\%) \\
\hline SVM (with ERBF kernel) & 92.26 & 90.41 & 91.33 \\
\hline GMM [47] & 90.44 & 88.58 & 89.51 \\
\hline k-NN [46] & 83.51 & 82.12 & 82.81 \\
\hline GMM = Gaussian mixture models; k-NN = k-nearest neighbor.
\end{tabular}

\subsection{The Robustness Evaluation of ACC System}

In this subsection, the robustness performance of the overall system of audio content classification (ACC) is evaluated against any variable noise-level conditions. Combined with VAD scheme and the hybrid-based SMD, the hierarchical architecture of audio content classification (ACC) can provide higher performance. First, in order to perform the cross-validation evaluation on the proposed ACC, mismatched training and testing data are required. A case for the model testing is on BBC radio and NHK/TTV news and training is on the well-known GTZAN database. Table 8 shows that the evaluation of cross-validation is tested on the on BBC radio and NHK/TTV news database when the models are trained on the GTZAN database. It was found that the proposed ACC system can successfully divide into speech, music, and noise/silence (the accuracy in speech is $91-92 \%$, Music $89-90 \%$, and Noise as $91-92 \%$ ) by using a hierarchical architecture, which combines hybrid- feature extraction and entropy-based VAD even in the cross-validation evaluation. In addition, we see that the comparison performance is almost robust against different training and the tested dataset. 
Table 8. Cross-validation evaluation on BBC radio and NHK/TTV news database using the models trained on the GTZAN database.

\begin{tabular}{ccccccc}
\hline \multirow{2}{*}{$\begin{array}{c}\text { Models } \\
\text { Trained on }\end{array}$} & \multicolumn{3}{c}{ BBC Radio Test } & \multicolumn{3}{c}{ NHK/TTV News Test } \\
\cline { 2 - 6 } & Sp (\%) & Mu (\%) & No (\%) & Sp (\%) & Mu (\%) & No (\%) \\
\hline GTZAN [75] & 91.5 & 90.4 & 92.7 & 92.4 & 89.2 & 91.7 \\
\hline Sp (\%) & speech (\%); Mu (\%) $=$ music (\%); No (\%) = noise/silence (\%).
\end{tabular}

Secondly, Table 9 illustrates that classification error rates on speech, music, and noise segments are reduced significantly to about a $6 \%$ error rate reduction after post-processing. The classification error rates, $C E R(\%)$, is defined below as:

$$
\operatorname{CER}(\%)=\frac{\text { num. of falsely class fied recordings }}{\text { total num. of testing audio recordings }} \times 100 \%
$$

Table 9. Comparison with/without post-processing.

\begin{tabular}{ccccc}
\hline $\begin{array}{c}\text { With/Without } \\
\text { Post-Processing Scheme }\end{array}$ & Speech CER (\%) & Music CER (\%) & Noise CER (\%) & Overall CER (\%) \\
\hline ACC & 17.43 & 18.62 & 15.73 & 17.26 \\
\hline $\begin{array}{c}\text { ACC + rule-based } \\
\text { post-processing }\end{array}$ & 11.96 & 12.73 & 10.89 & $\mathbf{1 1 . 8 6}$ \\
\hline
\end{tabular}

ACC $=$ audio content classification; $\mathrm{CER}=$ classification error rates

Finally, Table 10 shows that the performance of the proposed audio content classification (ACC) is robust against any variable noise-level conditions under the four types of background noise. Due to the proposed ACC, which is based on a hierarchical approach, it is firstly combined with the two schemes of entropy-based VAD and hybrid-based SMD for classifying the audio content. We can see that the experimental results of the ACC algorithm perform well at four noise types and levels, especially in realistic or poor SNR conditions. The main reason is attributed to the fact that the utilized entropy-based VAD segmentation can also perform successfully in real conditions with variable-noise levels and be excellently applied into the ACC application.

Table 10. The classification error rates of the proposed ACC algorithm under four noise types and levels.

\begin{tabular}{cccccccc}
\hline \multirow{2}{*}{$\begin{array}{c}\text { Noise } \\
\text { Type }\end{array}$} & $\begin{array}{c}\text { SNR } \\
\text { (dB) }\end{array}$ & Arti \#01 & Arti \#02 & Arti \#03 & Real \#01 & Real \#02 & Overall \\
\cline { 3 - 7 } CER (\%)
\end{tabular}




\subsection{Comparison of Other Classifier Systems}

Recently deep convolutional neural networks (deep CNNs) have been very successful at many tasks. The CNNs are designed and exploited to capture audio-related features for the problem of speech and music discrimination [83]. The results of the proposed hierarchical ACC method, along with the other compared methodologies including the deep CNN-based method on the publicly available audio datasets, are presented in Table 11. Our experimental setup was tested on a CPU $2.7 \mathrm{GHz}$ Intel Core i7 for the whole dataset.

Table 11. Comparisons between the proposed hierarchical method and other systems.

\begin{tabular}{ccccccccc}
\hline \multirow{2}{*}{ Dataset } & \multicolumn{2}{c}{ Proposed Method } & \multicolumn{2}{c}{ k-NN [84] } & \multicolumn{2}{c}{ GMM [85] } & \multicolumn{2}{c}{$\begin{array}{c}\text { Spectrogram-Based } \\
\text { CNN [83] }\end{array}$} \\
\cline { 2 - 9 } & Acc (\%) & T (min) & Acc (\%) & T (min) & Acc (\%) & T (min) & Acc (\%) & T (min) \\
\hline GTZAN [75] & 93.6 & 18.6 & 91.3 & 20.8 & 90.4 & 20.5 & 94.5 & 30.5 \\
LVLib-SMO [12] & 94.6 & 18.2 & 90.8 & 19.6 & 88.2 & 21.1 & 95.2 & 33.9 \\
RWC [77] & 94.2 & 19.6 & 92.2 & 21.8 & 89.1 & 20.8 & 95.8 & 35.1 \\
FMA-small [76] & 94.5 & 19.5 & 92.7 & 23.1 & 90.2 & 22.5 & 95.7 & 36.2 \\
\hline AVERAGE & 94.225 & 18.8 & 91.75 & 20.73 & 89.475 & 20.5 & 95.4 & 33.925 \\
\hline
\end{tabular}

$\mathrm{T}(\mathrm{min})=$ time in minutes, $\mathrm{k}-\mathrm{NN}=\mathrm{k}$-nearest neighbor, $\mathrm{GMM}=$ Gaussian mixture models.

The required computational demands are also evaluated in Table 11. We find that the spectrogrambased CNN achieves highest average accuracy with $95.4 \%$ under these four datasets. However, the computational time required is also the longest $(30.5 \mathrm{~min}$ to $36.2 \mathrm{~min}$ ) to complete the whole evaluation process for these four methods. The computational time includes spectrogram image transform and deep network size for learning features. Deep convolutional neural networks are computationally expensive compared to other systems. They enquire better computing hardware such as GPUs and neuromorphic chips to overcome this drawback. In addition, the CNN has the problem of overfitting and it mostly computationally expensive because it needs to take a large database for training. Compared to the spectrogram-based CNN, the proposed hierarchical ACC system using hybrid feature-based SMD and entropy-based VAD provides a great trade-off in terms of the computing complexity and accuracy. The results show that the execution time of the proposed hierarchical ACC system is almost only half of the spectrogram-based CNN method. Moreover, the average accuracy (with $94.225 \%$ ) of the proposed hierarchical ACC system is just a little smaller than that of the spectrogram-based CNN method. In actuality, the hierarchical classification has always been one of the great methodologies for audio content analysis. Moreover, a combination of voice activity detection (VAD), speech/music discrimination (SMD), and post-processing is novelty applied into the hierarchical classification. Especially, the voice activity detection (VAD) demonstrates a novel use of entropy. The proposed hierarchical classification system provides a reliable, stable, and low-performance architecture for the audio content analysis.

\section{Conclusions}

In this paper, we presented a new algorithm of audio content classification (ACC) for applications under a variable noise-level environment. A novel hierarchical scene of a three-stage scheme of the proposed ACC algorithm was described in detail for classifying audio stream into speech, music, and background noise. In addition, we introduced the hybrid-based feature, which investigates the use of 1D-subband energy information (1D-SEI) and 2D textural image information (2D-TII) as hybrid features to classify speech or music. It was found that using hybrid-based features can easily discriminate the noisy audio signal into speech and music. Further, the entropy-based VAD segment indeed provides high accuracy for application of the ACC. In summary, we conclude that the proposed ACC based on hybrid features SMD scheme and entropy-based VAD segment can achieve a low error value of below $13 \%$ at a low SNR and variable noise-level according to the above experimental results. It was 
shown that hybrid-based SMD and entropy-based VAD segments can be successfully applied into the system of audio content classification (ACC). The system was tested with different combinations of audio styles and different SNR levels. The experimental evaluations were also performed with real radio recordings from $\mathrm{BBC}, \mathrm{NHK}$, and TTV news.

In addition, the proposed hierarchical ACC system was compared with other systems on publicly available audio datasets. This paper proves that the hierarchical classification is one of the great methodologies for audio content analysis. Compared to the spectrogram-based CNN, the proposed hierarchical ACC system using hybrid feature-based SMD and entropy-based VAD can provide a great trade-off in terms of computing complexity and accuracy. Moreover, a combination of voice activity detection (VAD), speech/music discrimination (SMD), and post-processing is a novel idea, applied into the hierarchical classification. Especially, the voice activity detection (VAD) demonstrates a novel use of entropy. The proposed hierarchical classification system provides a reliable, stable, and low-performance architecture for contribution of audio content analysis.

In future work, the proposed ACC approach using hybrid-based manner will be appended to discriminate more audio types with lower SNR levels. In order to apply audio content retrieval, we will also focus on developing an effective scheme.

Author Contributions: The author proposed a combination of hierarchical classification including entropy-based voice activity detection (VAD), speech/music discrimination (SMD) and post-processing. Especially, the voice activity detection (VAD) demonstrates a novel use of entropy. The proposed hierarchical classification system provides a reliable, stable, and low-performance architecture for contribution of the audio content analysis.

Funding: This work was sponsored by the Ministry of Science and Technology, Taiwan (MOST106-2221-E-158-004, MOST107-2221-E-158-003 and MOST108-2221-E-158-003).

Acknowledgments: The author thank the Ministry of Science and Technology for their support with funding.

Conflicts of Interest: The author declares no conflict of interest.

\section{References}

1. Ong, W.Q.; Tan, A.W.C.; Vengadasalam, V.V.; Tan, C.H.; Ooi, T.H. Real-Time Robust Voice Activity Detection Using the Upper Envelope Weighted Entropy Measure and the Dual-Rate Adaptive Nonlinear Filter. Entropy 2017, 19, 487. [CrossRef]

2. Graf, S.; Herbig, T.; Buck, M.; Schmidt, G. Features for voice activity detection: A comparative analysis. EURASIP J. Adv. Signal Process. 2015, 2015, 91. [CrossRef]

3. Ghosh, P.K.; Tsiartas, A.; Narayanan, S. Robust voice activity detection using long-term signal variability. IEEE Trans. AudioSpeechand Lang. Process. 2010, 19, 600-613. [CrossRef]

4. Ma, Y.; Nishihara, A. Efficient voice activity detection algorithm using long-term spectral flatness measure. EURASIP J. AudioSpeechand Music Process. 2013, 2013, 87. [CrossRef]

5. Zhang, X.L.; Wu, J. Deep belief networks based voice activity detection. IEEE Trans. AudioSpeechand Lang. Process. 2013, 21, 697-710. [CrossRef]

6. Tsardoulias, E.; Thallas, A.G.; Symeonidis, A.L.; Mitkas, P.A. Improving Multilingual Interaction for Consumer Robots through Signal Enhancement in Multichannel Speech. J. Audio Eng. Soc. 2016, 64, 514-524. [CrossRef]

7. Dimoulas, C.A.; Symeonidis, A.L. Syncing Shared Multimedia through Audiovisual Bimodal Segmentation. IEEE Multimed. 2015, 22, 26-42. [CrossRef]

8. Tsipas, N.; Vrysis, L.; Dimoulas, C.; Papanikolaou, G. Efficient audio-driven multimedia indexing through similarity-based speech/music discrimination. Multimed. Tools Appl. 2017, 76, 25603-25621. [CrossRef]

9. Vrysis, L.; Tsipas, N.; Dimoulas, C.; Papanikolaou, G. Crowdsourcing audio semantics by means of hybrid bimodal segmentation with hierarchical classification. J. Audio Eng. Soc. 2016, 64, 1042-1054. [CrossRef]

10. Kotsakis, R.; Kalliris, G.; Dimoulas, C. Investigation of broadcast-audio semantic analysis scenarios employing radioprogramme-adaptive pattern classification. Speech Commun. 2012, 54, 743-762. [CrossRef]

11. Dimoulas, C.; Kalliris, G. Investigation of wavelet approaches for joint temporal, spectral and cepstral features in audio semantics. In Proceedings of the 134th AES Convention, Rome, Italy, 4-7 May 2013; pp. 509-518. 
12. Vrysis, L.; Tsipas, N.; Dimoulas, C.; Papanikolaou, G. Extending Temporal Feature Integration for Semantic Audio Analysis. In Proceedings of the 142th Audio Engineering Society (AES) Convention, Berlin, Germany, 20-23 May 2017.

13. Ntalampiras, S.; Potamitis, I.; Fakotakis, N. Exploiting temporal feature integration for generalized sound recognition. EURASIP J. Adv. Signal Process. 2009, 2009, 807162. [CrossRef]

14. Mathieu, B.; Essid, S.; Fillon, T.; Prado, J.; Richard, G. Yaafe, an easy to use and efficient audio feature extraction software. In Proceedings of the Eleventh International Society for Music Information Retrieval Conference (ISMIR 2010), Utrecht, The Netherlands, 9-13 August 2010; pp. 441-446.

15. Zahid, S.; Hussain, F.; Rashid, M.; Yousaf, M.H.; Habib, H.A. Optimized audio classification and segmentation algorithm by using ensemble methods. Math. Probl. Eng. 2015, 2015, 209814. [CrossRef]

16. Lerch, A. An Introduction to Audio Content Analysis: Applications in Signal Processing and Music Informatics; John Wiley \& Sons: Hoboken, NJ, USA, 2012.

17. Deng, L.; Hinton, G.; Kingsbury, B. New types of deep neural network learning for speech recognition and related applications: An overview. In Proceedings of the 2013 IEEE International Conference on Acoustics, Speech and Signal Processing, Vancouver, BC, Canada, 26-31 May 2013; pp. 8599-8603.

18. Sharan, R.V.; Moir, T.J. An overview of applications and advancements in automatic sound recognition. Neurocomputing 2016, 200, 22-34. [CrossRef]

19. Saunders, J. Real-time discrimination of broacast speech/music. In Proceedings of the 1996 IEEE International Conference on Acoustics, Speech, and Signal Processing Conference (ICASSP'96), Atlanta, GA, USA, 9 May 1996; pp. 993-996.

20. Scheirer, E.; Slaney, M. Construction and evaluation of a robust multifeature speech/music discriminator. In Proceedings of the 1997 IEEE International Conference on Acoustics, Speech, and Signal Processing (ICASSP'97), Munich, Germany, 21-24 April 1997; pp. 1331-1334.

21. El-Maleh, K.; Klein, M.; Petrucci, G.; Kabal, P. Speech/music discrimination for multimedia applications. In Proceedings of the 2000 IEEE International Conference on Acoustics, Speech, and Signal Processing (ICASSP'2000), Istanbul, Turkey, 5-9 June 2000; Volume 6, pp. 2445-2448.

22. Harb, H.; Chen, L. Robust speech music discrimination using spectrum's first order statistics and neural networks. In Proceedings of the Seventh International Symposium on Signal Processing and Its Applications, Paris, France, 4 July 2003; Volume 2, pp. 125-128.

23. Wang, W.Q.; Gao, W.; Ying, D.W. A fast and robust speech/music discrimination approach. In Proceedings of the 4th Pacific Rim Conference on Multimedia, Singapore, 15-18 December 2003; Volume 3, pp. 1325-1329.

24. Keum, J.S.; Lee, H.S. Speech/Music Discrimination using Spectral Peak Feature for Speaker Indexing. In Proceedings of the 2006 International Symposium on Intelligent Signal Processing and Communications (ISPACS ‘06), Tottori, Japan, 12-15 December 2006.

25. Eronen, A.; Klapuri, A. Musical instrument recognition using cepstral coefficients and temporal features. In Proceedings of the 2000 IEEE International Conference on Acoustics, Speech, and Signal Processing (Cat. No. 00CH37100), Istanbul, Turkey, 5-9 June 2000; Volume 752, pp. II753-II756.

26. Ghosal, A.; Chakraborty, R.; Chakraborty, R.; Haty, S.; Dhara, B.C.; Saha, S.K. Speech/music classification using occurrence pattern of zcr and ste. In Proceedings of the 2009 Third International Symposium on Intelligent Information Technology Application, Shanghai, China, 21-22 November 2009; pp. 435-438.

27. West, K.; Cox, S. Features and classifiers for the automatic classification of musical audio signals. In Proceedings of the 5th International Conference on Music Information Retrieval (ISMIR 2004), Barcelona, Spain, 10-14 October 2004; pp. 531-537.

28. Downie, J.S. The scientific evaluation of music information retrieval systems: Foundations and future. Comput. Music J. 2004, 28, 12-23. [CrossRef]

29. Beigi, H.S.; Maes, S.H.; Chaudhari, U.V.; Sorensen, J.S. A hierarchical approach to large-scale speaker recognition. In Proceedings of the Sixth European Conference on Speech Communication and Technology, Budapest, Hungary, 5-9 September 1999.

30. McKay, C.; Fujinaga, I. Automatic Genre Classification Using Large High-Level Musical Feature Sets. In Proceedings of the 5th International Conference on Music Information Retrieval (ISMIR 2004), Barcelona, Spain, 10-14 October 2004; pp. 525-530. 
31. West, K.; Cox, S. Finding An Optimal Segmentation for Audio Genre Classification. In Proceedings of the 6th International Conference on Music Information Retrieval (ISMIR 2005), London, UK, 11-15 September 2005; pp. 680-685.

32. Zhang, T.; Kuo, C.-C.J. Content-based classification and retrieval of audio. In Proceedings of the Advanced Signal Processing Algorithms, Architectures, and Implementations VIII, San Diego, CA, USA, 2 October 1998; pp. 432-443.

33. Ren, J.-M.; Chen, Z.-S.; Jang, J.-S.R. On the use of sequential patterns mining as temporal features for music genre classification. In Proceedings of the 2010 IEEE International Conference on Acoustics, Speech and Signal Processing, Dallas, TX, USA, 14-19 March 2010; pp. 2294-2297.

34. Ghosal, A.; Chakraborty, R.; Dhara, B.C.; Saha, S.K. Instrumental/song classification of music signal using ransac. In Proceedings of the 2011 3rd International Conference on Electronics Computer Technology, Kanyakumari, India, 8-10 April 2011; pp. 269-272.

35. Berenzweig, A.L.; Ellis, D.P. Locating singing voice segments within music signals. In Proceedings of the 2001 IEEE Workshop on the Applications of Signal Processing to Audio and Acoustics (Cat. No. 01TH8575), New Platz, NY, USA, 24-24 October 2001; pp. 119-122.

36. Zhang, T.; Kuo, C.C.J. Content-based Audio Classification and Retrieval for Audiovisual Data Parsing; Kluwer Academic: Los Angeles, CA, USA, 2001.

37. Zhang, T. Semi-automatic approach for music classification. In Proceedings of the SPIE Conference on Internet Multimedia Management Systems, Orlando, FL, USA, 26 November 2003; pp. 81-91.

38. Moore, B.; Glasberg, B.; Baer, T. A model for the prediction of thresholds, loudness and partial loudness. J. Audio Eng. Soc 1997, 45, 224-240.

39. Zwicker, E.; Fastl, H. Psychoacoustics: Facts and Models; Springer: Berlin, Germany, 1999.

40. Yu, G.; Slotine, J.-J. Audio classification from time frequency texture. In Proceedings of the 2009 IEEE International Conference on Acoustics, Speech and Signal Processing (ICASSP), Taipei, Taiwan, 19-24 April 2009; pp. 1677-1680.

41. Dennis, J.; Dat, T.; Li, H. Spectrogram image feature for sound event classification in mismatched conditions. IEEE Signal Process. Lett. 2011, 18, 130-133. [CrossRef]

42. Foote, J.T. Content-based retrieval of music and audio. In Proceedings of the Multimedia Storage and Archiving Systems II, Dallas, TX, USA, 6 October 1997; pp. 138-147.

43. Matityaho, B.; Furst, M. Classification of music type by a multilayer neural network. J. Acoust. Soc. Am. 1994, 95, 2959. [CrossRef]

44. Tsai, W.-H.; Bao, D.-F. Clustering music recordings based on genres. In Proceedings of the 2010 International Conference on Information Science and Applications, Seoul, Korea, 21-23 April 2010; pp. 1-5.

45. Zhang, T.; Kuo, C.C.J. Audio content analysis for online audiovisual data segmentation and classification. IEEE Trans. Speech Audio Process. 2001, 3, 27-36. [CrossRef]

46. Simsekli, U. Automatic music genre classification using bass lines. In Proceedings of the 2010 20th International Conference on Pattern Recognition, Istanbul, Turkey, 23-26 August 2010; pp. 4137-4140.

47. Tzanetakis, G.; Cook, P. Music genre classification of audio signals. IEEE Trans. Speech Audio Process. 2002, 10, 293-302. [CrossRef]

48. Costa, Y.M.G.; Oliveira, L.S.; Koreich, A.L.; Gouyon, F. Music genre recognition using spectrograms. In Proceedings of the 2011 18th International Conference on Systems, Signals and Image Processing, Sarajevo, Bosnia-Herzegovina, 16-18 June 2011; pp. 1-4.

49. Fernandez, F.; Chavez, F.; Alcala, R.; Herrera, F. Musical genre classification by means of fuzzy rule-based systems: A preliminary approach. In Proceedings of the 2011 IEEE Congress of Evolutionary Computation (CEC), New Orleans, LA, USA, 5-8 June 2011; pp. 2571-2577.

50. Pikrakis, A.; Theodoridis, S. Speech-music discrimination: A deep learning perspective. In Proceedings of the 2014 22nd European Signal Processing Conference (EUSIPCO), Lisbon, Portugal, 1-5 September 2014; pp. 616-620.

51. Lee, J.; Park, J.; Kim, K.L.; Nam, J. SampleCNN: End-to-End Deep Convolutional Neural Networks Using Very Small Filters for Music Classification. Appl. Sci. 2018, 8, 150. [CrossRef]

52. LeCun, Y.; Bengio, Y.; Hinton, G. Deep learning. Nature 2015, 521, 436. [CrossRef] [PubMed]

53. Wang, K.C. A Wavelet-Based Voice Activity Detection Algorithm in Variable-Level Noise Environment. WSEAS Trans. Comput. 2009, 6, 949-955. 
54. Laws, K.I. Textured image segmentation. Ph.D. Thesis, University of Southern California, Los Angles, CA, USA, 1980.

55. Wang, K.C. Time-Frequency Feature Representation Using Multi-Resolution Texture Analysis and Acoustic Activity Detector for Real-Life Speech Emotion Recognition. Sensors 2015, 15, 1458-1478. [CrossRef] [PubMed]

56. Wang, K.-C.; Yang, Y.-M.; Yang, Y.-R. Speech/music discrimination using hybrid-based feature extraction for audio data indexing. In Proceedings of the 2017 International Conference on System Science and Engineering, (ICSSE 2017), Ho Chi Minh City, Vietnam, 21-23 July 2017; pp. 515-519.

57. Neammalai, P.; Phimoltares, S.; Lursinsap, C. Speech and music classification using hybrid Form of spectrogram and Fourier transformation. In Proceedings of the Signal and Information Processing Association Annual Summit and Conference (APSIPA), 2014 Asia-Pacific, Siem Reap, Cambodia, 9-12 December 2014.

58. Yeh, J.H. Emotion Recognition from Mandarin Speech Signals. Master's Thesis, Tatung University, Taipei, Taiwan, 2004.

59. Junqua, J.C.; Mak, B.; Reaves, B. A Robust Algorithm for Word Boundary Detection in the Presence of Noise. IEEE Trans. Speech Audio Process. 1994, 2, 406-412. [CrossRef]

60. Lamel, L.; Labiner, L.; Rosenberg, A.; Wilpon, J. An Improved Endpoint Detector for Isolated Word Recognition. IEEE ASSP Magazine 1981, 29, 777-785. [CrossRef]

61. Wu, B.F.; Wang, K.C. A Robust Endpoint Detection Algorithm Based on the Adaptive Band-Partitioning Spectral Entropy in Adverse Environments. IEEE Trans. Speech Audio Process. 2005, 13, 762-775.

62. Wikipedia. Available online: https://en.wikipedia.org/wiki/Bark_scale (accessed on 29 January 2020).

63. Dimoulas, C.; Kalliris, G.; Papanikolaou, G.; Kalampakas, A. Novel wavelet domain Wiener filtering de-noising techniques: Application to bowel sounds captured by means of abdominal surface vibrations. Biomed. Signal Process. Control 2006, 1, 177-218. [CrossRef]

64. Wang, K.C. An Adaptive Wavelet-Based Denoising Algorithm for Enhancing Speech in Non-stationary Noise Environment. IEICE Trans. Inf. Syst. 2010, 93, 341-349. [CrossRef]

65. Wu, G.D.; Lin, C.T. Word boundary detection with mel-scale frequency bank in noise environment. IEEE Trans. Speech Audio Process. 2000, 8, 541-554.

66. Chen, S.H.; Wang, J.F. Speech enhancement using perceptual wavelet packet decomposition and teager energy operator. J. VLSI Signal Process. 2004, 36, 125-139. [CrossRef]

67. Costa, Y.; Oliveira, L.; Koerich, A.; Gouyon, F.; Martins, J. Music genre classification using LBP textural features. Signal Process. 2012, 92, 2723-2737. [CrossRef]

68. Costa, Y.; Oliveira, L.; Koerich, A.; Gouyon, F. Comparing textural features for music genre classification. In Proceedings of the 2012 International Joint Conference on Neural Networks (IJCNN), Brisbane, QLD, Australia, 10-15 June 2012; pp. 1-6.

69. Han, K.-P.; Park, Y.-S.; Jeon, S.-G.; Lee, G.-C.; Ha, Y.-H. Genre classification system of tv sound signals based on a spectrogram analysis. IEEE Trans. Consum. Electron. 1998, 44, 33-42.

70. Wang, K.C. The Feature Extraction Based on Texture Image Information for Emotion Sensing in Speech. Sensors 2014, 14, 16692-16714. [CrossRef] [PubMed]

71. Ding, P.; Chen, Z.; Liu, Y.; Xu, B. Asymmetrical support vector machines and applications in speech processing. In Proceedings of the 2002 IEEE International Conference on Acoustics, Speech, and Signal Processing, Orlando, FL, USA, 13-17 May 2002; pp. 73-76.

72. Ganapathiraju, A.; Hamaker, J.E.; Picone, J. Applications of support vector machines to speech recognition. IEEE Trans. Signal Process. 2004, 52, 2348-2355. [CrossRef]

73. Melgani, F.; Bruzzone, L. Classification of hyperspectral remote sensing images with support vector machines. IEEE Trans. Geosci. Remote Sens. 2004, 42, 1778-1790. [CrossRef]

74. Guo, G.; Li, S.Z. Content-based audio classification and retrieval by support vector machines. IEEE Trans. Neural Netw. 2003, 14, 209-215.

75. Gtzan Music Speech Dataset. Available online: http://marsyasweb.appspot.com/download/datasets/ (accessed on 30 July 2016).

76. Defferrard, M.; Benzi, K.; Vandergheynst, P.; Bresson, X. Fma: A dataset for music analysis. arXiv 2016, arXiv:1612.01840. 
77. Goto, M.; Hashiguchi, H.; Nishimura, T.; Oka, R. RWC Music Database: Popular, Classical and Jazz Music Databases. In Proceedings of the 3rd International Conference on Music Information Retrieval (ISMIR 2002), Paris, France, 13-17 October 2002; pp. 287-288.

78. Sohn, J.; Kim, N.S.; Sung, W. A statistical model-based voice activity detection. IEEE Signal Process. Lett. 1999, 16, 1-3. [CrossRef]

79. Ramirez, J.; Segura, J.C.; Benitez, C.; Garcia, L.; Rubio, A. Statistical voice activity detection using a multiple observation likelihood ratio test. IEEE Signal Process. Lett. 2005, 12, 689-692. [CrossRef]

80. ITU-T Recommendation G.729-Annex B, A silence compression scheme for G.729 optimized for terminals conforming to recommendation V.70. 1996.

81. ETSI EN 301708 recommendation, Voice activity detector (VAD) for adaptive multi-rate (AMR) speech traffic channels. 1999.

82. Tahmasbi, R.; Rezaei, S. A soft voice activity detection using GARCH filter and variance Gamma distribution. IEEE Trans Audio Speech Lang Process. 2007, 15, 1129-1134. [CrossRef]

83. Audio Classification Using CNN-An Experiment. Available online: https://medium.com/x8-the-aicommunity/audio-classification-using-cnn-coding-example-f9cbd272269e (accessed on 29 January 2020).

84. Lu, L.; Zhang, H.-J.; Jiang, H. Content analysis for audio classification and segmentation. IEEE Trans. Speech Audio Process. 2002, 10, 504-516. [CrossRef]

85. Minotto, V.P.; Lopes, C.B.; Scharcanski, J.; Jung, C.R.; Lee, B. Audiovisual voice activity detection based on microphone arrays and color information. IEEE J. Sel. Top. Signal Process. 2013, 7, 147-156. [CrossRef]

(C) 2020 by the author. Licensee MDPI, Basel, Switzerland. This article is an open access article distributed under the terms and conditions of the Creative Commons Attribution (CC BY) license (http://creativecommons.org/licenses/by/4.0/). 